\section{Coarsely sliced spectrum wavelength division multiplexing-passive optical networking for remote monitoring systems}

Frank S. Tsai, ${ }^{a}$ Matthias Gross, ${ }^{b}$ Douglas R. Jorgesen, ${ }^{a}$ Anis Husain, ${ }^{b}$ and Sadik C. Esener ${ }^{a}$

anniversity of California, San Diego, Department of Electrical and Computer Engineering, 9500 Gilman Drive, Mail Code 0405, La Jolla, California 92093-0407 E-mail: frank.sftsai@gmail.com

biva Corp, 6440 Lusk Blvd., Suite D 107, San Diego, California 92121

\begin{abstract}
We report the demonstration of the uplink of a low-cost passive optical network (PON), utilizing a superluminescent light emitting diode (SLED) as a broadband light source, a coarse wavelength division multiplexer (CWDM) to slice the spectrum into standard CWDM channels, and a reflective semiconductor optical amplifier (RSOA) as modulator and amplifier at the remote site. We demonstrate 2.5-Gbps transmission over $1 \mathrm{~km}$ of single-mode fiber for all four channels of the CWDM link (1511, 1531, 1551, and $1571 \mathrm{~nm}$ ), with the bit rate error (BER) of the system measured to be below $10^{-12}$. The main applications for this communication system are remote monitoring systems. (๑) 2010 Society of Photo-Optical Instrumentation Engineers.
\end{abstract}

[DOI: 10.1117/1.3454375]

Subject terms: remote sensing; remote monitoring; passive optical network; reflective semiconductor optical amplifier; coarse wavelength division multiplexing; superluminescent light emitting diodes.

Paper 091006LR received Dec. 31, 2009; revised manuscript received May 4, 2010; accepted for publication May 17, 2010; published online Jul. 1, 2010.

\section{Introduction}

In a remote monitoring system, data gathering sites are distributed over broad areas typically up to $1 \mathrm{~km}$ away from the central server station. The remote sites extract information from the environment, including high data rate information such as video. The extracted information is then transferred back to a central server station for analysis. One important remote monitoring application example is the mobile radar sensing technology, where the radar antennas are placed on radar trucks and distributed throughout a surveillance field. In a typical setting, radar trucks are distributed between $100 \mathrm{~m}$ to $1 \mathrm{~km}$ distance from the central processing truck, and the data rate is 2.5 Gbps per channel. ${ }^{1}$

Existing solutions for this type of communication system have significant drawbacks. For example, electronic cables are heavy and bulky, therefore fiber optics is preferred. Vertical cavity surface emitting laser (VCSEL) based optical transmitters have limited range and reliability, and suffer from wavelength shifts with temperature. ${ }^{2}$ Also,

0091-3286/2010/\$25.00 @ 2010 SPIE

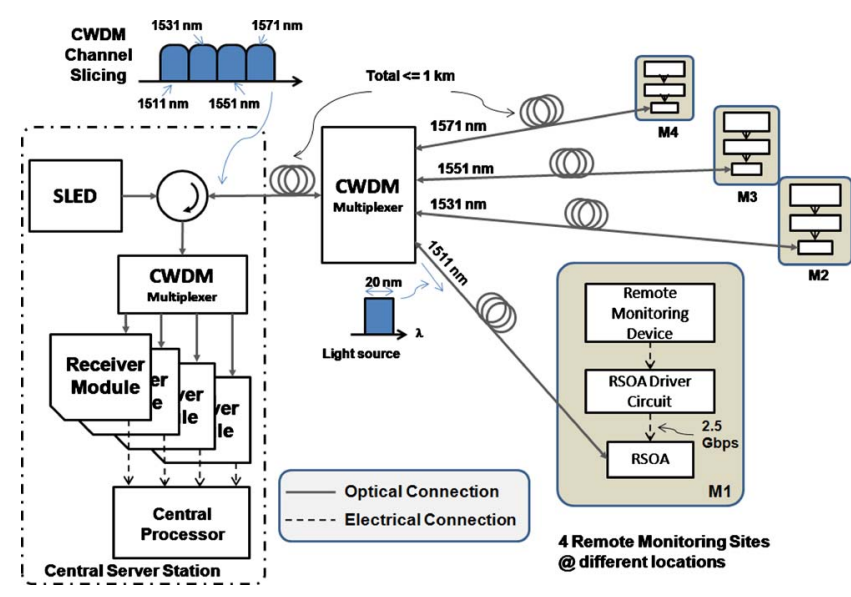

Fig. 1 Proposed architecture for the communication link.

dense wavelength division multiplexing passive optical network (DWDM-PON) is often too expensive. ${ }^{3}$

We propose a PON-like ${ }^{4,5}$ low-cost communication architecture utilizing superluminescent light emitting diodes (SLEDs), coarse wavelength division multiplexers (CWDMs), and reflective semiconductor amplifiers (RSOAs). The SLED is used as a low-cost broadband source, ${ }^{6}$ while the CWDM multiplexers are used to slice the entire spectrum into four different 20-nm-wide sources for each individual remote site. The utilization of a CWDM multiplexer instead of a DWDM multiplexer further reduces the cost and reduces the environmental (primarily temperature) constraints, both of which are important in a remote sensing application. Finally, the RSOA was chosen to modulate and amplify the remote data, since this device is relatively stable in a wide range of temperatures, ${ }^{7,8}$ reducing the requirements for environmental control at the remote site. However, commercially available RSOA are suitable for use at data rates up to only $1.25 \mathrm{Gbps}$; therefore, we utilized the pre-emphasis technique from microwave circuits to improve speed to $2.5 \mathrm{Gbps}$. We have demonstrated that the proposed architecture provides data transfer over $1-\mathrm{km}$ transmission distance for all four CWDM channels and promises bit error rate (BER) of better than $10^{-12}$ to enable Ethernet connections.

\section{Proposed Architecture}

The proposed PON architecture is shown in Fig. 1 and works as follows. the central office (central server station) is shown on the left side. Four remote monitoring sites (optical network units) are on the right side. The latter contain the RSOA as modulator and amplifier, RSOA driver circuit, and the monitoring devices (sensors). The light source for the optical link is a SLED placed in the central server station. This eliminates the need for multiple light sources, such as VCSELs or laser banks. Furthermore, the SLED is a robust device and not as sensitive to high temperatures as laser sources. This ensures a lower overall cost for the optical system due to relaxed temperature control requirements.

The SLED output is coupled via a circulator into a single-mode fiber. The remote end of this fiber is connected to a demultiplexer that slices the broadband optical signal into four standard 20-nm-wide CWDM channels. The 


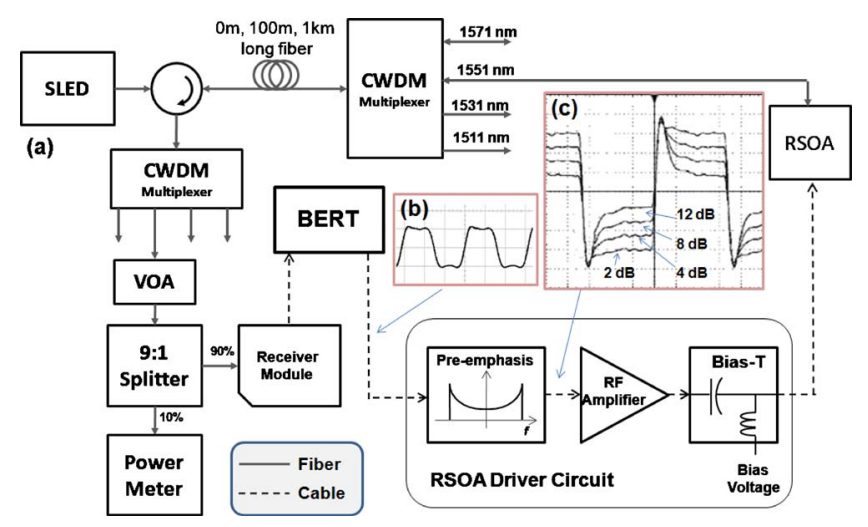

Fig. 2 (a) Constructed experimental system setup. (b) Waveform of signal from BERT. (c) Waveform with overshoot after pre-emphasis driver. Different levels of pre-emphasis have different output waveforms.

20-nm-wide optical sources are transmitted through a second fiber to the RSOAs, with total fiber lengths between SLED and RSOAs of up to $1 \mathrm{~km}$. The RSOA modulates the entire 20-nm-wide light source and reflects the signal back through the fiber, through the CWDM multiplexer, and back into the circulator. The circulator redirects the reflected signal into the receiver section of the central server station. The channels are separated again with another CWDM and fed into optical receivers. Finally, the data from all the remote sites are collectively analyzed by a central processor.

\section{Experimental Setup}

To demonstrate our proposed architecture, we built an experimental setup as shown in Fig. 2. For experimental purposes, we only populated one channel. The server site uses a SLED (DenseLight DL-BX9-CS5107A, Singapore) to provide the optical power needed for data transmission (8-mW total) and an APD-TIA receiver, which works at data rates up to 2.5 Gbps (OEMarket RX957, Sydney, Australia). The remote site contains the RSOA as the modulator (SOA-RL-OEC-1550 from CIP Technologies, IP switch, United Kingdom); the bit-error-rate tester as the data source [Anritsu (Richardson, Texas) 1632A BERT]; and a RSOA driver circuit, which is comprised of several key modules, as can be seen in Fig. 2(a).

The 3-dB frequency of the RSOA is only about $1.25 \mathrm{GHz}$. To achieve high quality data transmission at $2.5 \mathrm{Gbps}$, we used pre-emphasis, which is a technique that increases the amplitude of high frequency components of the signal, so that the entire system can achieve a higher bandwidth. The actual implementation of pre-emphasis is done in the time domain by adding an overshoot to the signal during transition; waveforms before and after application of pre-emphasis are shown in Figs. 2(b) and 2(c). Translated into frequency domain, the overshoot has the effect of amplifying the high frequency components. Overall, an RSOA is a low pass filter that rolls off around $1.25 \mathrm{GHz}$, but by integrating a pre-emphasis driver (which is a high pass filter) into the system, the overall frequency response is improved, allowing higher transmission data rates. In our RSOA driver circuit [Fig. 2(a)], we used a standard pre-emphasis driver (Maxim3982, Maxim, Sunny- vale, California) after which the signal is further amplified by an rf amplifier [Marki (Morgan Hill, California) A08058]. Finally, the amplified rf signal is combined with a $\mathrm{dc}$ bias current in the bias-tee (Marki BT-0018) and coupled into the RSOA.

To evaluate the sensitivity of the optical system, a variable optical attenuator (VOA) [Thorlabs (Trenton, New Jersey) VOA50-APC] is used to attenuate the optical signal, which is then coupled into the APD-TIA receiver. The electrical output signals are fed back into the BERT to analyze the BER.

\section{Experimental Results}

The pre-emphasis driver provides four different levels of pre-emphasis, as shown in Fig. 2(c). Therefore, we first determined the optimal level of pre-emphasis for the system. Then, since several components are wavelength dependent, we studied the performance of each individual channel. Finally, we evaluated the effect of long distance transmission for the system. The performance of the optical link was evaluated with a pseudorandom bit sequence (PRBS) pattern with a length of $2^{15}-1$ to represent radar data. The SLED output was measured as $-0.72,1.76,2.79$, and $1.55 \mathrm{dBm}$ in the $1511,1531,1551$, and $1571-\mathrm{nm}$ CWDM channels, respectively. Power received by the RSOA is $5 \mathrm{~dB}$ below those levels caused by attenuation in the system. The RSOA was operated without temperature control with a bias current of $60 \mathrm{~mA}$.

\subsection{Performance Comparison of Pre-Emphasis Levels}

The four different levels of pre-emphasis are 2, 4, 8, and $14 \mathrm{~dB}$. The performances of the four pre-emphasis levels were compared to a communication link with no preemphasis.

To analyze the performance of different pre-emphasis levels, the experimental system was set with a fiber length of $100 \mathrm{~m}$, and the 1551-nm channel was activated. The eye diagrams and the sensitivity measurement results are shown in Fig. 3(a): 8- and 4-dB pre-emphasis are very similar and show the best performance, with a sensitivity improvement of about $6 \mathrm{~dB}$ (at $10^{-9} \mathrm{BER}$ ) compared to the case without pre-emphasis. 2 - and $14-\mathrm{dB}$ pre-emphasis are only slightly better than no pre-emphasis. A pre-emphasis of 2-dB level is not sufficient, while a pre-emphasis of $14 \mathrm{~dB}$ overcompensates and leads to intersymbol interference (ISI), which can be seen as line doubling in the eye diagram. The sensitivity of the system using the optimal pre-emphasis level of $8 \mathrm{~dB}$ was measured as $-31.5 \mathrm{dBm}$.

\subsection{Performance Analysis of Different Channels}

Since 8-dB pre-emphasis provides the best performance, we kept this system parameter constant for further experiments to explore other system properties. The next key property we tested was the performance of the optical system for different CWDM channels: 1511, 1531, 1551, and $1571 \mathrm{~nm}$.

The eye diagrams of the channels and the sensitivity of the channels are shown in Fig. 3(b). We observed no noise floor for all channels and better than $10^{-12}$ BER perfor- 


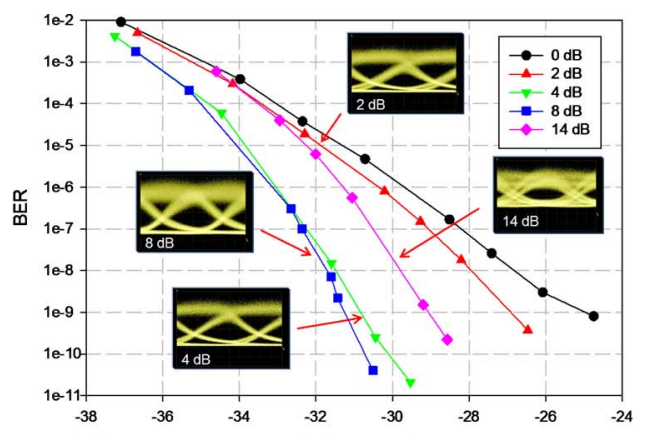

(a)

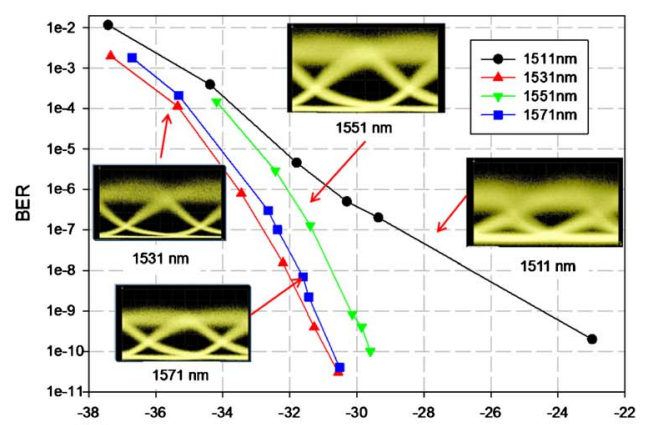

(b)

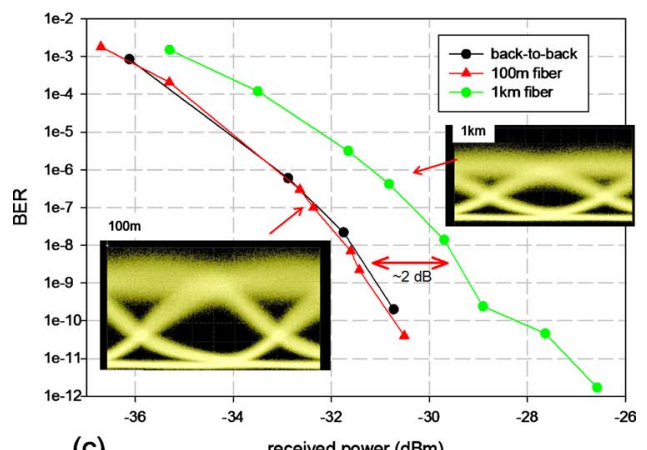

(c)

Fig. 3 Eye diagram and sensitivity measurement results for (a) different pre-emphasis levels, (b) different channels; and (c) different transmission lengths.

mance was demonstrated at $-10 \mathrm{dBm}$ received power by running the entire system for several hours with no errors detected.

The difference in the performance of the four channels can be explained with the wavelength-dependent SLED output and RSOA gain. Both are peaking around $1550 \mathrm{~nm}$ with a $3-\mathrm{dB}$ bandwidth of about $60 \mathrm{~nm}$. The performance drop in the 1511-nm channel is caused by the reduction of both SLED output and RSOA gain in that spectral region being the channel farthest from the maximum.

\subsection{Transmission over $1 \mathrm{~km}$ of Fiber}

With the performance of each channel identified, we measured the effect of fiber length on the performance of the communication link. The eye diagrams of the 1551-nm channel with $8-\mathrm{dB}$ pre-emphasis transmitted over $100 \mathrm{~m}$ and $1 \mathrm{~km}$ of fiber are shown in Fig. 3(c). While the eye is still open at $100 \mathrm{~m}$, it is beginning to close at $1 \mathrm{~km}$. This is mostly caused by chromatic dispersion. The total dispersion $\Delta t$ of a fiber link is $\Delta t=D \cdot \Delta \lambda \cdot L$, with $D$ being the disper- sion coefficient of the fiber, $\delta \lambda$ being the spectral bandwidth of the transmitted signal, and $L$ being the link length. For our experiments, we used standard a single-mode fiber with $D=17 \mathrm{ps} / \mathrm{nm} \times \mathrm{km}$. The bandwidth is determined by the $20 \mathrm{~nm}$ width of the CWDM channels. Therefore, the total dispersion of the $100-\mathrm{m}$ link is $34 \mathrm{ps}$, and for the $1-\mathrm{km}$ link it is 340 ps. By comparing to the eye width of about $400 \mathrm{ps}$ at $2.5 \mathrm{Gbps}$, we expect much stronger signal deterioration at $1 \mathrm{~km}$, as confirmed by the measured eye diagrams. Other effects that affect signal quality, e.g., attenuation, polarization mode dispersion (PMD), or nonlinearities, are not expected to influence this system and are not evident from the eye diagrams.

The BER performance measurement results are shown in Fig. 3(c). From the measurement data, we can see that the 100-m transmission system has similar properties compared to the back-to-back case. With 1-km transmission length, we measured a power penalty of $2 \mathrm{~dB}$.

\section{Conclusion}

We develop a working prototype for a low-cost, high-speed RSOA-based optical connector for remote monitoring applications. We demonstrate that a 2.5-Gbps optical fiber communication link can be achieved with a SLED as light source and a directly modulated RSOA for data transmission on a CWDM channel roster. The concept of preemphasis for driving the RSOA is introduced to boost the data rate from the manufacturer specified 1.25 Gbps to $2.5 \mathrm{Gbps}$. Low BER data transmission is demonstrated for all four CWDM channels with a power penalty in the 1511-nm channel due to the present bandwidth limitations of optical components. Transmission over $100 \mathrm{~m}$ and $1-\mathrm{km}$ distance is demonstrated, showing capability for in remote monitoring applications.

\section{Acknowledgements}

This project was funded by U.S. ARO under contract number W911NF-06-C-0085 via subcontract by Ziva Corporation.

\section{References}

1. M. Gross, H. Zhang, S. F. Tsai, P. Wen, S. esener, and A. Husain, "Active optical backplane/extenders for military systems," in Proc. Government Microcircuit Applications and Critical Technology Conf., Orlando, FL, March 16-19, 2009, paper 19-1 (2009).

2. S. Xie, R. W. Herrick, D. Chamberlin, S. J. Rosner, S. McHugo, G. Girolami, M. Mayonte, S. Kim, and W. Widjaja, "Failure mode analysis of oxide VCSELs in high humidity and high temperature," $J$. Lightwave Technol. 21(4), 1013-1019 (2003).

3. S.-M. Lee, K.-M. Choi, S.-G. Mun, J.-H. Moon, and C.-H. Lee, "Dense WDM-PON based on wavelength-locked Fabry-Perot laser diodes," IEEE Photonics Technol. Lett. 15(7) 1579-1581 (2005).

4. P. Healey, P. Townsend, C. Ford, L. Johnston, P. Townley, I. Lealman, L. Rivers, S. Perrin, and R. Moore, "Spectral slicing WDMPON using wavelength-seeded reflective SOAs," IEEE Electron. Lett., 37(15) 1181-1182 (2001).

5. F. Payoux, P. Chanclou, M. Moignard, and R. Brenot, "WDM PON based on spectral slicing and RSOA," ECOC (2005).

6. F. Payoux, P. Chanclou, and R. Brenot, "WDM PON with a single SLED seeding colorless RSOA-based OLT and ONUs," ECOC (2006).

7. K. Y. Cho, Y. Takushima, and Y. C. Chung, "Enhanced operating range of WDM PON implemented by using uncooled RSOAs," IEEE Photon. Technol. 20(18), 1536-1538 (2008).

8. A. Borghesani, I. F. Lealman, A. Poustie, D. W. Smith, and R. Wyatt, "High temperature, colourless operation of a reflective semiconductor optical amplifier for $2.5 \mathrm{Gbit} / \mathrm{s}$ upstream transmission in a WDMPON," ECOC, 641-642 (2007). 